\title{
Effect of Carthamus tinctorius L Extract on Diethylnitrosamine-Induced Liver Cirrhosis in Rats
}

\author{
Zhi-wei Hu and Wei-xing Wang* \\ Department of Hepatobiliary and Laparoscopic Surgery, Renmin Hospital of Wuhan University, Wuhan 430060, China
}

*For correspondence: Email: huzhiwei133494@163.com; Tel: +86 02788041911

Received: 5 February 2015

Revised accepted: 27 May 2015

\begin{abstract}
Purpose: To explore the effects of Carthamus tinctorius L. extract (CTLE) on diethylnitrosamine (DEN)induced liver cirrhosis in rats.

Methods: CTLE was obtained by extracting the dried Carthamus tinctorius L. in water. Liver cirrhosis was induced by injecting the rats with DEN once a week for 8 weeks. Following this treatment, clinical biochemical assessments, as well as oxidative stress test and histopathological examination were performed.

Results: Compared with the control group, plasma concentrations of alanine transaminase (ALT) and aspartate aminotransferase $(A S T)$ both decreased significantly $(p<0.01)$ after 8 weeks. The degree of liver fibrosis, cirrhosis and necrosis decreased in CTLE-treated rats. CTLE significantly inhibited malondialdehyde (MDA) and superoxide dismutases (SOD) in DEN-induced rat liver $(p<0.01)$ compared with control group.

Conclusion: CTLE has significant inhibitory effect on diethylnitrosamine-induced liver cirrhosis in rats, which can be developed for future clinical applications.
\end{abstract}

Keywords: Carthamus tinctorius L., Liver cirrhosis, Anti-oxidant, Apoptosis, Diethylnitrosamine

Tropical Journal of Pharmaceutical Research is indexed by Science Citation Index (SciSearch), Scopus, International Pharmaceutical Abstract, Chemical Abstracts, Embase, Index Copernicus, EBSCO, African Index Medicus, JournalSeek, Journal Citation Reports/Science Edition, Directory of Open Access Journals (DOAJ), African Journal Online, Bioline International, Open-J-Gate and Pharmacy Abstracts

\section{INTRODUCTION}

Liver fibrosis is a multi-step process resulting from various factors such as viral hepatitis, alcohol abuse, biliary atresia and hepatotoxins. Hepatic fibrosis is associated with apoptosis of activated hepatic stellate cells [1]. Characteristic of liver cirrhosis included cell viability and redox ratio decrease, reactive oxygen species formation, lipid peroxidation, DNA fragmentation, and formation of apoptotic bodies, which provided potential targets for therapy [2]. Inhibition of oxidative stress provoked and participated in the prevention of the progression of liver cirrhosis [3]. It has been found that DEN- induced liver cirrhosis in rats was similar to those of human cirrhosis $[4,5]$.

Modern pharmacological experiments have demonstrated that Carthamus tinctorius $L$ with its active compounds possesse wide-reaching biological activities, including dilated coronary artery, improved myocardial ischemia, modulated immune system, anticoagulation and antithrombosis, anti-oxidation, anti-aging, antihypoxia, anti-fatigue, anti-inflammation, antihepatic fibrosis, antitumor, analgesia, etc [6-11]. Due to its traditional use of Carthamus tinctorius $L$ in the prevention of liver cirrhosis [12], this study was performed on models of liver cirrhosis in rats. 


\section{EXPERIMENTAL}

\section{Material}

The herbal samples of Carthamus tinctorius $\mathrm{L}$ were collected from Bozhou City, Anhui Province in China in May 2014. Taxonomic identification of the plant was performed by Professor $\mathrm{Hu}$-lin Chen of Wuhan University in China. A voucher specimen of herbarium (no. CTL 201409024) was deposited in the College of Pharmacy, Wuhan University, China for future reference. The aqueous extract of CTL was obtained by steeping the dried Carthamus tinctorius $\mathrm{L}$ in water at $60{ }^{\circ} \mathrm{C}$ three times, each for one hour before first drying in an oven and then freezedrying the last extract thus obtained. One gram powder was equivalent to about $1.8 \mathrm{~g}$ crude samples. The yield was $55.56 \%$.

\section{Animals}

Male Wistar rats weighing 180 - $220 \mathrm{~g}$ were provided by the Experimental Animal Center of Hubei Province (Certificate no. SYXK 20070003). The animals had free access to food and water, and were allowed to acclimatize for at least one week before use. The rat experiment was approved by the Animal Care and Use Committee of Wuhan University (approval ref no. 20100906) and was carried out in compliance with Directive 2010/63/EU on the handling of animals used for scientific purposes [13].

\section{Animal groups}

The rats were randomly divided into 3 groups of ten rats each: Group I: injected with saline solution and oral saline solution after saline injection, used as normal group). Group II: injected with DEN $(70 \mathrm{mg} / 2 \mathrm{~mL} / \mathrm{kg}$ body weight/rat, once a week) and oral saline solution after DEN treatment for 8 weeks, used as a positive control group (Model group). Group III: injected with DEN $(70 \mathrm{mg} / 2 \mathrm{~mL} / \mathrm{kg}$ body weight/rat, once a week) and oral CTLE (40 $\mathrm{mg} / 10 \mathrm{~mL} / \mathrm{kg}$ body weight/rat, once every day) (CTLE group) after DEN treatment for 8 weeks.

\section{Biochemical analysis}

In the 4th and 8th week treatment, blood samples $(0.5 \mathrm{~mL})$ were collected into heparinized tubes from each rat by the puncture of the retro orbital sinus. Blood was immediately processed by centrifugation at $3500 \mathrm{~g}$ for $15 \mathrm{~min}$. Serum levels of alanine transaminase (ALT) and aspartate aminotransferase (AST) were measured by spectrophotometry, using commercially available kits (Nanjing Jiancheng Bioengineering Institute).

\section{Histological examination}

For the histopathological examination, the liver tissue was fixed in $10 \%$ formalin for paraffin embedding. Five- $\mu$ m-thick sections were prepared and stained with hematoxylin and eosin. It was randomly cut into 6 histological sections. Histopathology examination was completed using Nikon eclipse TE2000-U Microscope. Masson trichrome staining was used to identify collagen tissues.

\section{Determination of oxidative stress parameters in the liver tissue}

Rats were sacrificed and their livers were excised, rapidly washed and homogenized in ten volumes $(\mathrm{v} / \mathrm{w})$ of ice-cold saline solution. The homogenate was centrifuged at 3000 rpm for 10 min. Its supernatant was used as a total liver homogenized sample (10\% homogenates). The levels of malondialdehyde (MDA), a biomarker of lipid peroxidation, were determined spectrophotometrically by measuring thiobarbituric acid reactive substances (TBARS). $1 \mathrm{~mL}$ of $10 \%$ trichloroacetic acid and $1 \mathrm{~mL}$ of $0.67 \%$ thiobarbituric acid were added to $0.2 \mathrm{~mL}$ of the $10 \%$ homogenates of the tissue samples. The mixtures were incubated at $100{ }^{\circ} \mathrm{C}$ for $15 \mathrm{~min}$. After cooling and centrifugation, the supernatant was aspirated and its absorbance at 532 (A532) and $600 \mathrm{~nm}$ (A600) determined using water blank. MDA concentration ( $\mu \mathrm{mol} / \mathrm{L})$ was computed according to the reference [14]. SOD activity was measured by spectrophotometry, using commercially available SOD kit, A001-1 (Nanjing Jiancheng Bioengineering Institute).

\section{Statistical analysis}

Data are presented as mean \pm standard deviation (SD) and were analyzed statistically by one-way ANOVA followed by Tukey's multiple comparison using SPSS 16.0 software for Windows. Differences were considered statistically significant at $p<0.05$.

\section{RESULTS}

\section{Fibrosis and cirrhosis}

With the weekly DEN-injection, the body weight of rats dropped gradually. DEN administration induced marked increases in the activity of serum ALT and AST $(p<0.01)$ compared with the normal group. CTLE ameliorated the 
increase of ALT and AST. After the 8th week DEN-injection, liver cirrhosis formed in the control and CTLE groups. Plasma concentrations of ALT and AST in the 8th-week control group significantly decreased compared with that in the 4th-week control group $(p<0.05)$, but they were still higher than that in the normal group. Compared with control group,plasma concentrations of ALT and AST both decreased significantly $(p<0.01)$ in the 8th-week (Tables 1 and 2).

\section{Histological features}

Blue regions in Fig $1 \mathrm{~b}$ and $\mathrm{c}$ indicated $\mathrm{a}$ significant level of fibrosis in the DEN-induced rats. As Fig 1 shows, there were overt cirrhosis and nodule formation with fibrosis in DENinduced rats. The degrees of liver fibrosis, cirrhosis and necrosis were mild scattered in CTLE-treated rats and confluent zonal in nontreated group. Meanwhile, scores for fatty infiltration were mild in CTLE group and moderate in control group.

\section{Effect of CTLE on MDA and SOD levels in liver cirrhosis rats}

MDA, presented statistically significantly increased levels in DEN-induced rats $(p<0.01)$. CTLE significantly inhibited MDA formation in DEN-induced rat liver $(p<0.01)$. Conversely, the levels of SOD were significantly increased in the livers of rats receiving CTLE administration compared with non-treated cirrhosis group (Table $3)$.

Table 3: Effect of CTLE on MDA and SOD contents in rat liver

\begin{tabular}{lcc} 
Group & $\begin{array}{c}\text { MDA } \\
\text { (umol/L) }\end{array}$ & $\begin{array}{c}\text { SOD } \\
\text { (U/mg protein) }\end{array}$ \\
\hline Normal & $0.08 \pm 0.03^{\star *}$ & $0.82 \pm 0.12^{\star *}$ \\
Control & $0.32 \pm 0.02$ & $0.46 \pm 0.06$ \\
CTLE & $0.13 \pm 0.02^{\wedge}$ & $0.73 \pm 0.044^{\wedge}$ \\
\hline${ }^{*} P<0.05$, & ${ }^{* *} p<0.01$ vs. control group
\end{tabular}

Table 1: Effect of CTLE on serum ALT (U/L)

\begin{tabular}{cccc}
\hline Groups & 0 week & 4 weeks & 8 weeks \\
\hline Normal & $101.3 \pm 3.4$ & $99.26 \pm 3.6$ & $100.6 \pm 3.5$ \\
Control & $102.5 \pm 3.3$ & $523.1 \pm 12.4^{\star \pi}$ & $238.4 \pm 9.6^{\star}$ \\
CTLE & $102.8 \pm 3.4$ & $152.6 \pm 5.7 \Delta \Delta$ & $137.3 \pm 4.4 \Delta \Delta$ \\
\hline$P<0.05, \quad p<0.01$ vs. normal group; $\Delta p<0.05, \quad \Delta \Delta p<0.01$ vs. control group
\end{tabular}

Table 2: Effect of CTLE on serum AST (U/L)

\begin{tabular}{cccc}
\hline Groups & 0 week & 4 weeks & 8 weeks \\
\hline Normal & $108.6 \pm 4.2$ & $104.4 \pm 3.8$ & $106.1 \pm 3.2$ \\
Control & $106.4 \pm 4.5$ & $347.6 \pm 10.4{ }^{\wedge}$ & $198.5 \pm 5.3$ \\
CTLE & $101.5 \pm 4.7$ & $134.7 \pm 6.2 \Delta \Delta$ & $152.1 \pm 3.4 \Delta \Delta$ \\
\hline${ }^{* *} P<0.05,{ }^{* *} p<0.01$ vs normal group; $\Delta p<0.05$ & $\Delta \Delta p<0.01$ vs. control group
\end{tabular}
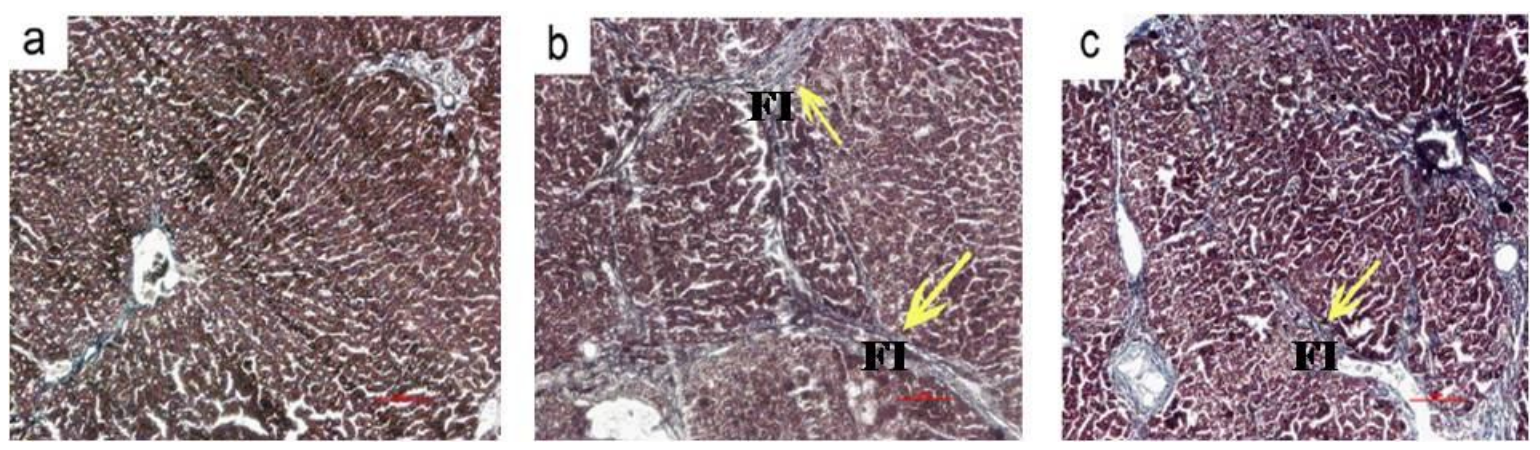

Fig 1: Masson trichrome histological slides of rat liver tissues. a: normal group; b: control group; c: CTLE group(Original magnification 100). Fl: fibrosis

\section{DISCUSSION}

Recently, many researchers have focused on the anti-fibrosis properties of herb medicines.In our research, oral administration of CTLE showed improvement in liver fibrosis and cirrhosis induced by DEN. The mechanisms that participated in the induction of the fibrotic process include necrosis, apoptosis [15], inflammatory reactions and the activation of 
hepatic stellate cells [16]. MDA, as an end product of lipid peroxidation, significantly increased in DEN-induced liver cirrhosis in rats [17]. CTLE remarkably inhibited MDA formation and increase the enzyme activity of SOD in DENinduced rats. CTLE inhibited DEN-induced fibrosis process through the anti-oxidant activity. CTLE reduced the extent of DNA damage and serum levels of ALT and AST at the 4th and the 8th weeks compared with non-treated DENinduced rats. All these demonstrated that CTLE participated in the attenuation of apoptosis, oxidative stress and DNA fragment in fibrotic liver. CTLE may be a potential antifibrotic agent in the future.

In the present study, CTLE was effective in the treatment of chemically induced liver fibrosis in rats. Their anti-fibrosis mechanisms that participated in the inhibition of the fibrotic process included anti-oxidant, anti-apoptosis, and metabolic disturbance in DEN-induced liver tissues of rats.

\section{CONCLUSION}

CTLE has significant inhibitory effect on diethylnitrosamine-induced liver cirrhosis in rats, which can be developed for future clinical applications.

\section{REFERENCES}

1. Tang X, Yang J, Li J. Accelerative effect of leflunomide on recovery from hepatic fibrosis involves TRAILmediated hepatic stellate cell apoptosis. Life Sci 2009; 84: 552-557.

2. Lakshmi Devi, S, Anuradha CV. Mitochondrial damage, cytotoxicity and apoptosis in iron-potentiated alcoholic liver fibrosis: amelioration by taurine. Amino Acids 2010; 38: 869-879.

3. Lv P, Luo HS, Zhou XP, Chireyath Paul S, Xiao YJ, Si $X M$, Liu SQ. Thalidomide prevents rat liver cirrhosis via inhibition of oxidative stress. Pathol. Res. Pract. 2006; 202: 777-788.

4. Gang Z, Qi Q, Jing C, Wang C. Measuring microenvironment mechanical stress of rat liver during diethylnitrosamine induced hepatocarcinogenesis by atomic force microscope. Microsc. Res. Tech. 2009; 72: 672-678.

5. Jin N, Deng J, Chadashvili T, Zhang Y, Guo Y, Zhang Z, Yang G.Y, Omary RA, Larson AC. Carbogen gas- challenge BOLD MR imaging in a rat model of diethylnitrosamine-induced liver fibrosis. Radiology 2010; 254: 129-137.

6. Xiao PG. Modern Chinese Material Medica, Vol 2. Beijing: Chemical Industrial Press: 2002; $p 178$.

7. Cheng JX, Zhu ZJ, Liang G, He MZ, Cai FS, Zhang MA. The effects of Ligusticu Chuanxiong Hort and Carthamus Tinctorius $L$ on the immune system in rats. Pract. Clin. J. Integr. Tradit. Chin. West. Med. 1993; 6: 261-262.

8. Li YH, Wang NS. Antithrombotic effects of Danggui, Honghua and potential drug interaction with clopidogrel. J.Ethnopharmacol. 2010; 128: 623-628.

9. Wang $Y$, Chen $P$, Tang $C Y$, Wang $Y, L i ~ Y Z$, Zhang $H$. Antinociceptive and anti-inflammatory activities of extract and two isolated flavonoids of Carthamus tinctorius L. J Ethnopharmacol. 2014; 151: 944-950.

10. Wang CC, Choy CS, Liu YH, Cheah KP. Protective effect of dried Safflower petal aqueous extract and its main constituent,carthamus yellow, agains tlipopolysaccharide-Induced inflammation in RAW 264.7 macrophages. J. Sci. Food Agric.2011; 91: 218-225.

11. Hiramatsu $M$, Takahashi $T$, Komatsu $M$, Kido $T$, Kasahara M. Antioxidant and neuroprotective activities of Mogami-benibana (Safflower, Carthamus tinctorius Linne). Neurochem. Res. 2009; 34: 795805.

12. Zou TY. Clinical study of safflower injection combined with Astragalus injection treatment in patients with decompensated cirrhosis. Chin J Ethnomed and Ethnopharm. 2008; 10: 45-47.

13. European Commission [homepage on the internet]. Directive 2010/63/EU on the protection of animals used for scientific purposes [cited 2013 Jan 16]. Available from:http://ec.europa.eu/environment/ chemicals/lab_animals/legislation_en.htm.

14. Yoon SY, Kim CY, Han HJ. Protective effect of ischemic postconditioning against hepatic ischemic reperfusion injury in rat liver. Ann Surg Treat Res. 2015; 88: 241 245.

15. Lu B, Xu, Y, Xu L, Cong $X$, Yin L, Li H, Peng J. Mechanism investigation of dioscin against CCl4induced acute liver damage in mice. Environ. Toxicol. Pharmacol. 2012; 34: 127-135.

16. Friedman, SL. Mechanisms of disease: mechanisms of hepatic fibrosis and therapeutic implications. Nat. Clin. Pract. 2004; 1: 98-105.

17. Tan $Y$, Yin $P$, Tang $L$, Xing $W$, Huang $Q$, Cao $D$, Zhao $X$, Wang $W$. Metabolomics study of stepwise hepatocarcinogenesis from the model rats to patients: potential biomarkers effective for small hepatocellular carcinoma diagnosis. Mol. Cell. Proteom 2012; 11: 257-259. 